\title{
Validity and Reliability of the Interpersonal Sensitivity/Privileged Self Scale: Solving a New Type of Depression
}

\author{
Itsuki Yamakawa', Masaki Muranaka1, Shinji Sakamoto² \\ ${ }^{1}$ Graduate School of Literature and Social Sciences, Nihon University, Tokyo, Japan \\ ${ }^{2}$ College of Humanities and Sciences, Nihon University, Tokyo, Japan \\ Email: yamakawa.itsuki@gmail.com
}

Received 22 May 2015; accepted 23 June 2015; published 26 June 2015

Copyright (C) 2015 by authors and Scientific Research Publishing Inc.

This work is licensed under the Creative Commons Attribution International License (CC BY).

http://creativecommons.org/licenses/by/4.0/

(c) () D Den Access

\begin{abstract}
Although "modern-type" depression (MTD), having different features than melancholic-type depression, has become problematic in Japan especially since the late 1990s, there are few psychological studies of MTD. Sakamoto, Muranaka, and Yamakawa (2014) proposed a psychological framework depicting the onset of MTD, and coined a new concept of interpersonal sensitivity/ privileged self, which is assumed to be a vulnerability factor for MTD. In the present study, we examined the validity and reliability of the Interpersonal Sensitivity/Privileged Self Scale (IPS; Muranaka, Yamakawa, \& Sakamoto, 2014), which has two superordinate factors, namely Interpersonal Sensitivity (IS: 16 items) and Privileged Self (PS: 9 items). Because MTD is presumed to overlap with atypical depression, it was expected that the IPS score in an atypical depression group would be higher than in a melancholic depression group, and the IPS score would be positively correlated with the depressive symptoms. Participants were 112 (70 male, 42 female) Japanese undergraduates, aged 18 - 25 years $(M=19.19, S D=2.07)$. Cronbach's $\alpha$ of the IPS was 0.90 (IS: $\alpha=0.91$, PS: $\alpha=0.72$ ). As we predicted, the IPS, IS, and PS were positively correlated with depressive symptoms. ANOVAs revealed significant differences in the scores of the IPS, IS, and PS among depression types. Post-hoc analyses showed that scores for anatypical depression group were significantly higher than those for a melancholic group and an unclassified-type group. These results support our prediction that IPS scores manifest differently in MTD and melancholic depression; validity and reliability of the IPS were confirmed.
\end{abstract}

\section{Keywords}

Depression, Interpersonal Sensitivity, Personality, Atypical, Japanese Undergraduates 


\section{Introduction}

In this study we examined the validity and reliability of a newly developed scale, the Interpersonal Sensitivity/ Privileged Self Scale (IPS). The new concept of interpersonal sensitivity/privileged self was coined in order to explain the psychological aspects of “modern-type” depression (MTD), which has been observed in Japan, especially after 1990 (Tarumi \& Kanba, 2005). As many Japanese psychiatrists have pointed out (e.g., Tarumi, 2005; Kato et al., 2011), MTD has different features from those of traditional-type depression (i.e., melancholic depression; Table 1). For instance, compared with melancholic depression, the symptoms of MTD are mild.

While people with melancholic depression are considered sympathetically, and viewed as honest, or hard working, those with MTD are considered selfish, immature, or sluggish. Because melancholic depression has been regarded as a textbook example of depression in Japan, MTD is confounding not only for professionals, but also for laypersons.

Moreover, MTD is not limited to Japan. Using case vignette methods, Kato et al. (2011) showed that similar cases to MTD were reported from other Asian counties or regions (i.e., Bangladesh, India, Iran, Korea, Taiwan, Thailand) as well as Australia and USA. In addition, some Japanese psychiatrists (Abe, 2012; Natsume, 2012)

Table 1. A comparison of melancholic depression and modern-type depression in Japan.

\begin{tabular}{|c|c|c|}
\hline & Melancholic depression & Modern type depression \\
\hline Ages & Middle age (born before 1970) & Young (born after 1970) \\
\hline \multirow[t]{4}{*}{ Temperaments } & Shuchaku-kishitsu (Shimoda, 1932) & Student apathy (Walters, 1961) \\
\hline & Typusmelancholicus (Tellenbach, 1961) & Withdrawal neurosis (Kasahara \& Kimura, 1975) \\
\hline & Avoidant-type depression (Hirose, 1977) & $\begin{array}{l}\text { Modern-type of depression } \\
\text { (Matsunami \& Yamashita, 1991) }\end{array}$ \\
\hline & & Immature-type depression (Abe et al., 1995) \\
\hline \multirow[t]{6}{*}{ Characters } & Attachment to rules & Attachment to self without roles \\
\hline & Love for order, models & Feel distressed against rules/order \\
\hline & Sympathetic & Negative feelings toward order or model \\
\hline & Obsessive & Vague sense of almighty \\
\hline & Honest & Not a hard worker to begin with \\
\hline & Hard worker & \\
\hline \multirow[t]{3}{*}{ Symptoms } & Agitation or retardation & Fatigue and not feel good enough \\
\hline & Exhaustion and guilt & Avoidance and blame others \\
\hline & Well-prepared suicide & Impulsive suicidal action \\
\hline \multirow[t]{3}{*}{ Therapeutic relationship } & Resist to diagnosis of depression & Cooperate with diagnosis of depression \\
\hline & When recovered, learn from experience of depression & Tend to check depressive symptoms \\
\hline & & $\begin{array}{l}\text { Hard to depart from diagnosis } \\
\text { (like to stay in depression) }\end{array}$ \\
\hline \multirow[t]{2}{*}{ Drug response } & Usually good & Partial response \\
\hline & Complete remission & \\
\hline \multirow[t]{2}{*}{ Cognition } & $\begin{array}{l}\text { I as a teacher to I as a "man who } \\
\text { experienced depression" }\end{array}$ & Life style or depression \\
\hline & & Hard to depart from "I suffer from depression" \\
\hline \multirow[t]{2}{*}{ Prognosis } & Good response to rest and antidepressants & $\begin{array}{l}\text { Become chronically ill only with rest } \\
\text { and antidepressants }\end{array}$ \\
\hline & Ambivalent about change of environment & Change of environment rapidly improves symptoms \\
\hline
\end{tabular}

Note: Cited and adapted from Tarumi (2005) and Kato et al. (2011). 
have stated that the symptoms of atypical depression (e.g., mood reactivity, interpersonal rejection sensitivity) depicted in the Diagnostic and Statistical Manual of Mental Disorders (DSM IV-TR; American Psychiatric Association, 2000) overlap with those of MTD. Thus, psychological studies of MTD are important in countries other than Japan.

Although psychological studies of MTD may be universally important, MTD is not officially defined (Note 1; Japanese Society of Mood Disorders, 2012), and thus the available psychological studies are limited. In initiating studies of MTD, Muranaka, Yamakawa, and Sakamoto (2015) began by collecting descriptions of the psychological characteristics of MTD. First, they found 14 Japanese books about MTD written by psychiatrists, which were intended for general readership, and extracted 105 items relating to psychological characteristics of MTD from those books. These 105 items were analyzed using the KJ method (Kawakita, 1967; Note 2), which resulted in the consolidation of 105 items into 64. The next step involved the use of expert judgments, so as to further reduce the number of the items and to examine the common understanding of MTD among clinicians. After adding four items relating to melancholic-type depression, a total of 68 items were presented to 13 psychiatrists, each of whom had more than eight years of experience in the field, and 11 clinical psychologists, each of whom reported more than 10 years of experience in the field. They were asked to assess whether each description was observed only among people with MTD and not those with other types of depression, including the melancholic type. Consequently, it appeared that the psychological characteristics of people with MTD could be summarized by the following three aspects (Table 2): interpersonal sensitivity, privileged self, and insisting on depression.

On the basis of this result, Sakamoto, Muranaka, and Yamakawa (2014) coined the term "interpersonal sensitivity/privileged self" and proposed a psychological framework depicting the onset of MTD (Note 3). Interpersonal sensitivity is defined as having both of the following two aspects: (a) a tendency to be excessively worried about other's negative evaluations, and (b) a tendency to excessively react (i.e., overreact) to such negative evaluations. Privileged self is defined as a tendency to pursue one's pleasure prior at the expense of maintaining harmony with others. The combination of interpersonal sensitivity and privileged self is thought to be a vulnerability factor for MTD. It is assumed that people with high interpersonal sensitivity tend to be shocked and become depressed after receiving negative evaluations by others who have significant influence on them (e.g., their superiors). In experiencing a depressed mood, people high in privileged self may prioritize pursuing their pleasure and be motivated to lessen their unpleasant experiences (e.g., depressed mood, negative evaluations from others), so that they may contradict or avoid the people who evaluated them negatively. In contrast, people low in privileged self are thought to be concerned about their relationships with the people around them (e.g., superiors and colleagues in their office), and may be motivated to make efforts to retrieve their honor (e.g.,

\section{Table 2. Three aspects of psychological characteristics of MTD.}

Interpersonal sensitivity

1 Blame others when one is hurt by what somebody else has said

2 Lack of self-accusation, being overly punitive, and opting to put the blame on others

3 Blame others when making a mistake

4 A strong avoidance tendency

Privileged self

5 Prioritize one's feelings and circumstances

6 Find it difficult to do what he/she does not want to do, although it is easy to do what he/she wants to do

7 Dislikes being bound by norms

Insisting on depression

8 Obtain a medical certificate indicating depression

9 Insist on "being depressed" or suggesting that it "may be depression"

Note: Cited and adapted from Muranaka et al. (2015). 
recover losses) or to blame themselves and apologize for not performing their duties (e.g., duties as an office worker). This may be the case in melancholic depression.

In order to examine Sakamoto et al.'s (2014) hypothetical model empirically, the Interpersonal Sensitivity/Privileged Self Scale (IPS) was developed (Table 3; Muranaka, Yamakawa, \& Sakamoto, 2014). The outline

\section{Table 3. The final 25 items of the Interpersonal Sensitivity/Privileged Self Scale.}

\begin{tabular}{|c|c|}
\hline Interpersor & ensitivity \\
\hline Evaluat & apprehension \\
\hline 1 & I worry that I may be criticized for my words and actions \\
\hline 2 & I don't worry much about how those around me see me $\mathrm{a}^{\mathrm{a}}$ \\
\hline 5 & I am afraid that those around me will have a poor image of me. \\
\hline 9 & I don't worry too much even if I'm criticized by those around me $\mathrm{a}^{\mathrm{a}}$ \\
\hline 11 & I worry that those around me may complain about me or look at me strangely \\
\hline 12 & I have no confidence unless my good aspects are praised, recognized, or accepted \\
\hline 15 & I tend to fluctuate between gladness and sadness in response to what those around me think of me \\
\hline 18 & I can’t act normally if I think about how I will appear to those around me \\
\hline 20 & If my words or actions aren't well thought of by those around me, I can't help worrying about it \\
\hline
\end{tabular}

Overreaction to negative feedback

3 I think I tend to get very hurt if I'm criticized or scolded

4 If I'm upset by those around me, I am not able to put it easily out of my mind

13 If I'm criticized by those around me, it nags at me for a long time

19 I am able to quickly move on even if I'm criticized by those around $\mathrm{me}^{\mathrm{a}}$

22 If I'm criticized by those around me, I can't forget about it

Avoidance

23 I think I actively take on challenges even if I'm likely to make a mistake ${ }^{a}$

25 I think I often do nothing to avoid making mistakes

Privileged self

Sense of victimization

6 I think my problems are caused by circumstances and people around me

8 I think there are a lot of mean people around me

10 I think I'm constantly being blamed for no reason

24 I think I am being treated unfairly in my everyday life

Self-righteousness

14 I think I often feel irritated if I'm criticized or admonished

16 I can’t stand people who don't accept my opinion

21 I get in a bad mood if things don't go my way

Results dependence

$7 \quad$ I think people's value is determined by their results and achievements

17 I think that results and achievements are unrelated to my own value ${ }^{\mathrm{a}}$

Note: Each number indicates the presentation order in the IPS. ${ }^{\mathrm{a}}$ Reverse-scored item. 
is described briefly below. First, on the basis of Muranaka et al.'s (2015) work, candidate items were generated and included in the first set comprising the psychological characteristics of MTD in the newly constructed scale. Subsequently, through surveys using undergraduates, a final list of 25 descriptions of personality traits related to MTD was selected. Exploratory factor analyses extracted six factors, which were summarized into two superordinate factors through higher-order factor analysis. The first superordinate factor was Interpersonal Sensitivity (IS; 16 items), which represents intense reactions to negative evaluation by others. IS comprises three subordinate factors: evaluation apprehension, over-reaction to negative feedback, and avoidance. The second superordinate factor was Privileged Self (PS; 9 items), which measures the degree to which people think that they should be regarded highly. PS also comprises three subordinate factors: sense of victimization, self-righteousness, and results dependence.

Here, we report the results obtained during the initial study, which evaluated the validity and reliability of the IPS. Based on the previous discussion, it was hypothesized that the IPS score would be positively correlated with depressive symptoms. We also speculated that IPS scores would manifest differently in MTD and melancholic depression. Specifically, the IPS total score and PS subscale score of people with MTD would be higher than those of people with melancholic depression.

\section{Method}

\subsection{Sample Size Design}

To detect a mean difference in IPS among depression subtypes, the sample size was calculated on the basis of previous study. Boyce, Hickie, Parker, Mitchell, Wilhelm, and Brodaty (1993) examined the mean differences of the Interpersonal Sensitivity Measure (Boyce \& Parker, 1989), which is a psychological scale measuring personality traits among people with melancholic depression, non-melancholic depression, and controls, by using one-way analysis of variance (Note 4). Because their study yielded an effect size of $f=0.28$, we estimated that the IPS would have a similar effect size. Hence, approximately 130 participants were required for a two-tailed significance level of $5 \%$ and power of $80 \%$.

\subsection{Participants and Procedure}

Questionnaires were administered to participants during a lecture period. The participants were 129 (75 male, 46 female, 8 unknown; age range 18 - 26 years) Japanese undergraduates enrolled in an introductory psychology course at a large private university near Tokyo. The students were briefly oriented to the broad aims of the study and then asked to complete the questionnaire on their own. All the questionnaires were filled in anonymously. The data of 17 participants were omitted from the analysis because they made incomplete responses to questions. The final sample thus was 112 (70 male, 42 female; age range 18 - 25 years, $M=19.19, S D=2.07$ ).

\subsection{Measures}

The Interpersonal Sensitivity/Privileged Self Scale (IPS) was developed to measure the personality features of individuals with MTD, and is assumed to indicate vulnerability factors for MTD. In the present study, to examine the convergent and divergent validity of the IPS, we administered the Global Scale for Depression (GSD; Fukunishi \& Fukunishi, 2012). The GSD is a 30-item scale divided into two parts, that is, the assessment of depressive symptoms and distinction between depression subtypes (i.e., melancholic, atypical, and unidentified). The first part of this scale (GSD-1; 17 items) measures the level of depressive symptoms. The degree of the distress associated with each item is rated on a 3-point scale ranging from 1 (not at all) to 3 (frequently). The second part (GSD-2; 13 items) measures the type of depression affecting an individual. Each item is rated on a 3-point scale ranging from -1 to 1 (range: -13 -13). A score of -3 or less denotes the atypical type of depression, whereas a score of 3 or more denotes melancholic depression. Scores between -3 and 3 are categorized into an unidentified group. Since previous studies (Abe, 2012; Natsume, 2012) have stated that atypical depression presumably overlaps with MTD, the GSD serves adequately to compare scores of the IPS in MTD with melancholic depression. Fukunishi and Fukunishi (2012) verified the concurrent validity and reliability of the GSD in a clinical sample of 412 Japanese individuals, who were diagnosed with a mood disorder. The GSD-1 positively correlates with the Zung Self-Rating Depression Scale (Zung, 1965; $r=0.481, p<0.05$ ), the Center for Epidemiologic Studies Depression Scale (Radloff, 1977; $r=0.521, p<0.05$ ), Hamilton Rating Scale for Depression 
(Hamilton, 1960; $r=0.509, p<0.05$ ), and Montgomery Asberg Depression Rating Scale (Montgomery \& Asberg, 1979; $r=0.474, p<0.05$ ). With respect to reliability, the GSD exhibited an alpha coefficient value of around 0.90; in our survey, the GSD-1 yielded $\alpha=0.86$.

\subsection{Statistical Analyses}

R for Windows version 3.2.0 (R Development Core Team, 2012) and "anovakun version 4.6.2", an ANOVA function that runs on $\mathrm{R}$ software, were used for all statistical analyses. The significance level was set at $\alpha=0.05$ for the analysis of variance, and post-hoc comparisons were conducted using a Games-Howell procedure.

\section{Results}

\subsection{Reliability}

The IPS yielded $\alpha=0.90$; the IS part of the scale yielded $\alpha=0.91$ and the PS part yielded $\alpha=0.72$.

\subsection{Validity}

The criterion-related validity of the IPS was estimated through Pearson's correlation coefficients. There was a positive correlation between the IPS and the GSD-1 ( $r=0.31$, 95\%CI [0.13, 0.47], $p<0.001)$. Examining the correlation of the two subscales of the IPS with the GSD-1, we obtained similar results: IS and PS positively correlated with the GSD-1 (IS: $r=0.22$ [0.03, 0.39], $p=0.023$; PS: $r=0.39$ [0.22, 0.54], $p<0.001$ ). In addition, the two subscales were positively correlated each other $(r=0.47$ [0.31, 0.60], $p<0.001)$.

For the ANOVA, participants were classified into three types of depression based on their total scores on the GSD-2. The mean score on the GSD-2 was 0.40 , the standard deviation was 2.83 , and the scores ranged from -7 to 8 . Of these participants, 18 (8 male, 10 female) were classified as having the features of atypical depression, 24 (13 male, 11 female) as melancholic, and 70 (49 male, 21 female) were classified into neither category. There was no significant sex difference in those subtypes $\left(\chi^{2}(2)=4.89, p=0.09\right)$.

One-way ANOVAs were conducted on the IPS total score as well as two subscales scores (IS, PS; Table 4). The results of the ANOVA on the total means obtained for the IPS showed significant differences between the depression types $\left(F(2,109)=4.32, p=0.02, \eta^{2}=0.07,95 \%\right.$ CI $\left.[0.002,0.171]\right)$. Post-hoc comparisons revealed that the atypical-type group obtained a significantly higher IPS score than the unclassified-type group and melancholic-type group. Similarly, the mean PS score differed significantly between the depression subtypes ( $F$ (2, $\left.109)=3.46, p=0.03, \eta^{2}=0.06,[0,0.15]\right)$. Post-hoc tests found that the atypical-type group had a significantly higher PS score than the unclassified- and the melancholic-type groups. Although there were no significant group differences on the IS score $\left(F(2,109)=2.72, p=0.07, \eta^{2}=0.05,[0,0.13]\right)$, post-hoc analysis revealed significant differences among depression types; As well as the IPS and PS scores, the mean IS score of the atypical-type group was higher than that of other two groups. However, the unclassified group and melancholic-type

Table 4. Descriptive statistics for all variables across the three groups.

\begin{tabular}{|c|c|c|c|c|c|c|c|c|c|c|c|c|}
\hline \multirow[b]{3}{*}{ Measure } & \multicolumn{4}{|c|}{ Atypical type $(n=18)$} & \multicolumn{4}{|c|}{ Melancholic type $(n=24)$} & \multicolumn{4}{|c|}{ Unclassified $(n=70)$} \\
\hline & \multicolumn{4}{|c|}{$95 \%$ CI } & \multicolumn{4}{|c|}{$95 \%$ CI } & \multirow[b]{2}{*}{$M$} & \multirow[b]{2}{*}{$S D$} & \multicolumn{2}{|c|}{$95 \%$ CI } \\
\hline & $M$ & $S D$ & $L L$ & $U L$ & $M$ & $S D$ & $L L$ & $U L$ & & & $L L$ & $U L$ \\
\hline IPS $^{\mathrm{a}}$ & 83.33 & 10.05 & 78.19 & 88.48 & 70.58 & 12.47 & 65.21 & 75.96 & 72.89 & 16.42 & 68.94 & 76.83 \\
\hline IS $^{\mathrm{b}}$ & 58.89 & 8.01 & 54.79 & 62.99 & 50.71 & 10.12 & 46.34 & 55.07 & 52.27 & 13.19 & 49.10 & 55.44 \\
\hline $\mathrm{PS}^{\mathrm{c}}$ & 24.39 & 5.05 & 21.81 & 26.97 & 20.62 & 4.35 & 18.75 & 22.50 & 20.91 & 5.49 & 19.60 & 22.23 \\
\hline GSD-1 ${ }^{\mathrm{d}}$ & 31.83 & 3.64 & 29.97 & 33.70 & 29.62 & 6.80 & 26.69 & 32.56 & 26.49 & 6.09 & 25.02 & 27.95 \\
\hline GSD-2 ${ }^{\mathrm{e}}$ & -4.06 & 0.97 & -4.55 & -3.56 & 4.33 & 1.14 & 3.84 & 4.83 & 0.20 & 1.30 & -0.11 & 0.51 \\
\hline
\end{tabular}

Note: CI = confidence interval; $L L=$ lower limit, $U L=$ upper limit, IPS = Interpersonal Sensitivity/Privileged Self Scale, IS = Interpersonal Sensitivity, PS = Privileged Self, GSD-1 = the first part of Global Scale for Depression, GSD-2 = the second part of Global Scale for Depression. ${ }^{\mathrm{a}}$ The possi-

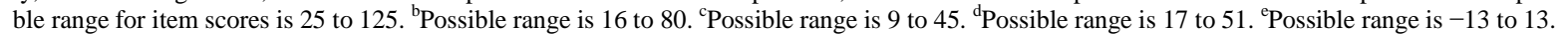


group did not significantly differ in their IS score.

\section{Discussion}

The IPS was developed to assess the personality features of individuals with MTD. In relation to this, the aim of the present study was to confirm the reliability and validity of the IPS. Consistent with our prediction, the IPS demonstrated excellent reliability and strong convergent and discriminative validity with regard to the following two points. First, the IPS, including its two subscales, positively correlated with depressive symptoms. These correlations imply that people displaying high interpersonal sensitivity/privileged self are vulnerable to depression. Second, IPS scores significantly differed according to depression type. Specifically, the total IPS score obtained by the atypical-depression group was higher than those of the melancholic type and unidentified groups. Similarly, the PS score obtained by the atypical-depression group was higher than those obtained by the unidentified and melancholic-type groups. Since atypical depression is presumed to overlap with MTD (Abe, 2012; Natsume, 2012), this result supports our prediction that IPS scores manifest differently in MTD and melancholic depression. Although the main effect of depression type was not significant for IS scores, multiple comparisons detected significant differences between the atypical type and other two types. The lower effect size of IS compared to total IPS and PS may be the reason for the nonsignificant result. That is, because IS included some items describing features that are common to MTD and melancholic depression, the mean difference was reduced in IS.

In order to examine the causal relationship between interpersonal sensitivity/privileged self and the onset of MTD, future studies will need to address the following three issues: cross-cultural aspects, the case of office workers, and the influence of health education regarding depression. First, we believe that cross-cultural studies are necessary. Although Kato et al. (2011) reported that MTD seemed prevalent in many countries other than Japan, as Tarumi and Kanba (2005) suggested (Note 5), it is possible that the Japanese socio-cultural background contributes to the development of MTD. Therefore cross-cultural studies are needed to investigate the hypothesis that the Japanese socio-cultural environment may heighten the influence of interpersonal sensitivity/privileged self on the onset of MTD. Second, because MTD has become a subject of discussion in company organizations in Japan, studies involving office workers must be conducted. In the work place where an individual is required to play a social role as a worker, the conflict between the social role and the private self, which is independent of the social role may be more likely to occur. Thus, we may be able to easily observe the relationship among interpersonal sensitivity/privileged self and MTD in a sample of office workers. The last issue is the influence of health education regarding depression on MTD. As Kanba (2011) suggested, health education regarding depression has been leading to the destigmatization of depression. It has become easier for people who are suffering from maladaptation to complain of their distress in the form of depression. Therefore, it is hypothesized that less stigma about depression may influence people who are high in privileged self, and encourage them to complain of depression more easily. To better understand the psychological mechanism of MTD, we believe that psychological studies of MTD are important; IPS may be useful in such studies.

Finally, some limitations must be considered. The present study is cross-sectional, thus the causal relationship between interpersonal sensitivity/privileged self and MTD was not investigated. Longitudinal studies are needed to examine the vulnerability hypothesis that interpersonal sensitivity/privileged self interacts with negative evaluations from others and leads to the onset of MTD. Moreover, it is still unknown whether the same results would be replicated in clinical samples as well as in other countries. Despite these limitations, the present study showed the reliability and validity of the Interpersonal Sensitivity/Privileged Self Scale, which may be one tool by which to solve the puzzling problem of MTD.

\section{Acknowledgements}

This work was supported by JSPS KAKENHI Grant Number 25380854.

\section{References}

Abe, T. (2012). Changeable and Immature "New Type Depression”: Consideration on Depression Today from the Psychopathological Viewpoint. Japanese Journal of Clinical Psychology, 12, 469-474. (In Japanese)

Abe, T., Otsuka, K., Nagano, M., Kato, S., \& Miyamoto, T. (1995). A Consideration on “Immature Type of Depression”: Premorbid Personalities and Clinical Pictures of Depression from the Structural-Dynamic Viewpoint (W. Janzarik). Japanese Journal of Psychopathology, 16, 239-248. (In Japanese) 
American Psychiatric Association (2000). Diagnostic and Statistical Manual of Mental Disorders (4th ed. Text Revision). Washington, DC: American Psychiatric Association.

Boyce, P., \& Parker, G. (1989). Development of a Scale to Measure Interpersonal Sensitivity. Australasian Psychiatry, 23, 341-351.

Boyce, P., Hickie, I., Parker, G., Mitchell, P., Wilhelm, K., \& Brodaty, H. (1993). Specificity of Interpersonal Sensitivity to Non-Melancholic Depression. Journal of Affective Disorders, 27, 101-105. http://dx.doi.org/10.1016/0165-0327(93)90082-U

Fukunishi, I., \& Fukunishi, A. (2012). The Manual for the Global Scale of Depression. Aoyama: Aoyama Psychological Clinical Education Center.

Hamilton, M. (1960). A Rating Scale for Depression. Journal of Neurology, Neurosurgery, and Psychiatry, $23,56-62$. http://dx.doi.org/10.1136/jnnp.23.1.56

Hirose, T. (1977). Tohi-gatautsu-byonitsuite. In T. Miyamoto (Ed.), Psychopathology of Manic_Depressive Illness 2 (pp. 61-86). Tokyo: Kobundo.

Japanese Society of Mood Disorders, Committee for the Creation of Treatment Guidelines on Mood Disorders (2012). Treatment Guidelines II, Major Depressive Disorder 2012 ver. 1.

http://www.secretariat.ne.jp/jsmd/mood_disorder/img/130924.pdf

Kanba, S. (2011). The Modern Society and Depression (1). SAISHIN IGAKU, 66, 1046-1048.

Kasahara, Y., \& Kimura, B. (1975). Clinical Classification of Depression. Seishin Shinkeigaku Zasshi, 77, 715-735.

Kato, T., Shinfuku, N., Fujisawa, D., Tateno, M., Ishida, T., Akiyama, T. et al. (2011). Introducing the Concept of Modern Depression in Japan; an International Case Vignette Survey. Journal of Affective Disorders, 135, 66-76. http://dx.doi.org/10.1016/j.jad.2011.06.030

Kawakita, J. (1967). Method of Conception. Tokyo: Chuo-shinsho.

Matsunami, K., \& Yamashita, Y. (1991). Social Changes and Depression. Japanese Journal of Social Psychiatry, 14, 193200.

Montgomery, S. A., \& Asberg, M. (1979). A New Depression Scale Designed to be Sensitive to Change. The British Journal of Psychiatry, 134, 382-389. http://dx.doi.org/10.1192/bjp.134.4.382

Muranaka, M., Yamakawa, I., \& Sakamoto, S. (2014). Development of the Interpersonal Sensitivity/Privileged Self Scale (1): Relationship with Severity and Type of Depression. The 11th Annual Meeting of the Japanese Society of Mood Disorders, Hiroshima, 18-19 July 2014, 186.

Muranaka, M., Yamakawa, I., \& Sakamoto, S. (2015). How Do Clinicians Define “Modern Type Depression”? Extraction from Books and the Survey to Clinicians. Psychological Research, Nihon University, 36, 44-51.

Natsume, M. (2012). Awareness and Support by Referring to the Case of Mental Health (7) Modern type depression Part 1 Definition and Hardship in the Workplace, Industrial Safety and Health, 63, 697-699.

R Development Core Team (2012). R: A Language and Environment for Statistical Computing. Vienna: R foundation for Statistical Computing. http://www.R-project.org/

Radloff, L. S. (1977). The CES-D Scale: A Self-Report Depression Scale for Research in the General Population. Applied Psychological Measurement, 1, 385-401. http://dx.doi.org/10.1177/014662167700100306

Sakamoto, S., Muranaka, M., \& Yamakawa, I. (2014). "Self” in the Interface of Social and Clinical PsychologyThrough the Discussion of Modern Type Depression. Japanese Psychological Review, 57, 405-429.

Shimoda, M. (1932). On the Treatment of Involutional Depression in my Department. Taiwan Igaku Zasshi (Formosa Med J), 31, 113-115.

Tarumi, S. (2005). The “New” Variant of Depression: The Dysthymic Type. Japanese Journal of Clinical Psychiatry, 34, 687-694.

Tarumi, S., \& Kanba, S. (2005). The Dysthymic Type of Depression: An Essay on Socio-Cultural Aspects of Depression. Japanese Bulletin of Social Psychiatry, 13, 129-136.

Tellenbach, H. (1961). Melancholie. Berlin: Springer. http://dx.doi.org/10.1007/978-3-662-12458-1

Walters Jr., P. A. (1961) Student Apathy. In G. B. Blame Jr., \& C. C. McArthur (Eds.), Emotional Problems of the Students (pp. 129-147). New York: Appleton-Century-Crofts.

Yamakawa, I., \& Sakamoto, S. (2015). Insisting on Depression, but Not Showing Symptoms: A Japanese Study of Excuse-Making. International Journal of Psychological Studies, 7, 146-154. http://dx.doi.org/10.5539/ijps.v7n2p146

Yoshino, S. (2009). Is it Really “Depressive Disorder”? The Countermeasures against "Working Depression” in Companies are Full of Mistakes. Tokyo: Kodansya.

Zung, W. W. K. (1965). A Self Rating Depression Scale Archives of General Psychiatry, 12, 63-70.

http://dx.doi.org/10.1001/archpsyc.1965.01720310065008 


\section{Notes}

Note 1. MTD is considered inclusive of several subtypes of depression, which were primarily proposed by Japanese psychiatrists: "Taikyakushinkei-sho" (withdrawal neurosis; Kasahara \& Kimura, 1975), "Tohi-gatautsu-byo" (avoidant type of depression; Hirose, 1977), "Gendaigatautsu-byo” (modern type of depression; Matsunami \& Yamashita, 1991), "Mizyuku-gatautsu-byo" (immature type of depression; Abe et al., 1995), and "Dysthymia-gatautsu-byo" (dysthymic type of depression; Tarumi, 2005; Tarumi \& Kanba, 2005).

Note 2. The KJ method was developed for creative thinking and involves the use of cards to create a conceptual map during brainstorming. In Japan, the KJ method is sometimes used for qualitative studies in academic as well as practical settings.

Note 3. While the two aspects of psychological characteristics of MTD (Table 2), interpersonal sensitivity and privileged self, are main factors that contribute to the onset of MTD, the remaining factor, insisting on depression, is thought to be a behavioral phenotype that is caused by the above two aspects. For example, office workers who are high in interpersonal sensitivity are more likely to feel depressed when they make mistakes in their job and receive negative evaluations from their superiors than those who are low in interpersonal sensitivity. Sakamoto et al. inferred that people having high privileged self would insist on depression in such a situation, and claim that their depression is caused by their superior's behavior (e.g., "They injured me mentally, so I have become depressed"). Insisting on depression may benefit them in other ways. Because suffering from depression is thought to serve as a reason for evading one's duty (Yoshino, 2009), insisting on depression may function as an excuse for not making efforts to recover their mistakes (Yamakawa \& Sakamoto, 2015). Thus, insisting on depression may be explained by interpersonal sensitivity and privileged self.

Note 4. Boyce and Parker (1989) have also proposed "interpersonal sensitivity" as a vulnerable personality style associated with depressive disorder (i.e., melancholic depression). Subsequently, Boyce et al. (1993) found that interpersonal sensitivity is also related to the development of non-melancholic type depression. Muranaka et al. (2015) discussed the differences between their concept and that of Boyce and Parker (1989); generally interpersonal sensitivity is common to both modern-type and melancholic-type depression. However, it appears that how people with MTD behave or respond in situations where they are criticized differs from people with melancholic depression.

Note 5. Tarumi and Kanba (2005) suggested that socio-economical changes may cause MTD. For instance, in Japan, the role of occupation was more prevalent in the 1970s than currently. However, with the passage of time, the importance of social order has waned and such social roles have been disrupted. Consequently, a kind of overprotected individualism has become the framework within Japan, and the number of Japanese people who are attached to themselves without a social role has increased (Tarumi \& Kanba, 2005). These changes may cause Japanese people to protect themselves from environmental stress by claiming that they are suffering from depression (Yoshino, 2009). MTD is considered an indication of such behavior. 